\title{
"Yes to discuss different models of care between primary care physicians and diabetes-practice nurses, but not to complete implementation yet": Explorative qualitative study at diabetes clinics in primary health care centres in Muscat, Oman
}

\author{
Kamila Al-Alawi*1, Johansson Helene ${ }^{2}$ \\ ${ }^{1}$ Department of Training and Studies, Royal Hospital, Ministry of Health, Muscat, Oman \\ ${ }^{2}$ Department of Epidemiology and Global Health, Umea University, Umea, Sweden
}

Received: August 6, 2019

DOI: $10.5430 /$ ijh.v6n1p72
Accepted: February 19, 2020

Online Published: March 9, 2020

URL: https://doi.org/10.5430/ijh.v6n1p72

\begin{abstract}
Background: Globally, many models of care through which the way health services are delivered have been adopted within team-based primary health care. Although these models have aimed to solve some of the health care challenges related to physician's shortages in clinics and further acceptance of non-physician-led clinics, their application is usually determined by a range of factors, such as preparedness of the health care providers, preparedness of patients and support from higher authorities. Objective: The study was designed to explore health care providers' perceptions for changes in models of care in diabetes clinics at primary health care in Muscat, Oman.

Methods: A total of 27 semi-structured interviews were conducted with health care providers involved in diabetes clinics at five purposively selected primary health care centres in Muscat. The interviewees included the core members of the diabetes management team and other supportive members available at the centres, and were of mixed genders, nationalities and professions. Qualitative thematic analysis was applied.

Results: The analysis resulted in one main theme, which captured positive responses towards task-sharing model, but revealed worries and requirements for complete implementation. Nurses' competences and diabetic patients' acceptance were among the main concerns. Health care providers revealed that for complete implementation of the model, nurses' involvement in the team could be improved through updating their knowledge and through the provision of support from higher authorities, while diabetic patients' acceptance could be improved through understanding of their perceived knowledge towards the model which could promote nurse-led clinics.

Conclusion: Task-sharing within the discussed possibilities could provide many positive outcomes and a rewarding future for diabetes clinics at primary health care centres. Omani culture could play a role in its implementation; therefore, if successful implementation is desired, carefully considered steps must be applied by the government and the community.
\end{abstract}

Key Words: Models of care, Primary health care providers, Primary health care, Type 2 diabetes

*Correspondence: Kamila Al-Alawi; Email: kamila_alalawi@yahoo.com; Address: PO Box 5, Post Code 138, The Wave, Seeb, Muscat, Oman. 


\section{BACKGROUND}

Extensive discussion has been handled about the way health services should be delivered as health workforce development in response to Human Immunodeficiency Virus epidemics in Africa. ${ }^{[1-3]}$ The discussion was related to their effectiveness in providing primary health care services and providing long-term solutions to shortages in physicians. The literature discussed substitution of doctors by nurses at primary health care in response to population ageing, rising patient expectations, and a continuous shift in care from hospitals to the community. ${ }^{[4]}$ At the same time, the supply of physicians is constrained by various factors and there is increasing pressure to contain costs. Shifting care from physicians to nurses within team-based approach is one possible response to these challenges, maintaining the team concept and providing the required care. The expectation is that nurse-doctor substitution, which is the eventual outcome to the team-based approach through different models of care, will reduce costs and physicians' workload while maintaining quality of care. ${ }^{[4]}$

The literature provides several health care models between primary health care providers. ${ }^{[2,5,6]}$ The most common examples are task-sharing, task-shifting and task-replacement. Task-sharing is the process of enabling healthcare professionals - such as nurses, midwives, clinical officers and community health workers - to provide clinical tasks with close supervision from physicians. For example; encouraging the diabetes practice nurses to perform an electrocardiography for the diabetic patients with interpretations from the physicians. Task-shifting is described as a situation where a task normally performed by a physician is transferred completely to a health professional with a different or lower level of education and training, or to a person specifically trained to perform limited tasks only. For example; encouraging the diabetes practice nurses to perform diabetic foot examination independently without physicians' supervision. Taskreplacement is the process where a particular task is replaced by a totally different task in the clinic such as replacing the sole physicians' decision approach by physician-patient counterpart decision approach by empowering patients' selfeducation and management.

The Type 2 diabetes epidemic in the Gulf countries, including Oman, is considered a subject of importance and makes continual service demands in a situation of persistent shortage of physicians. Therefore, a lot should be learned from settings where different models of care have been successfully applied and have achieved good results with solid evidencebased examples. ${ }^{[7]}$ The diabetes clinics in primary health care are trying to operationalize team-based approach. The core diabetes team in Oman consists of a physician, a nurse, a

Published by Sciedu Press dietician, and a health educator. ${ }^{[8]}$ The team-based approach is constructed on the available national evidence-based guidelines, which were developed based on the current research related to the prevention and treatment of diabetes, supported by the International Diabetes Federation, ${ }^{[9,10]}$ the American Association of Clinical Endocrinology and the American College of Endocrinology and Diabetes Management Guidelines. ${ }^{[11]}$

The clinics in primary health care in Oman are the levels where the diseases are first suspected, diagnosed, treated and followed up including diabetes. Therefore, health care providers in diabetes clinics at primary health care centres are among the essential factors of health care. Accordingly, they become key determinants of health in the population due to their first encounter with the patients, their primary knowledge they pass on to them and their management. In addition, other factors, such as socioeconomic status and environmental and behavioural factors could be added too. ${ }^{[12]}$ The way the health system is organized, managed, and regulated affects health care providers' performance. ${ }^{[13]}$ Relatedly, it affects patients' health outcomes and the service in terms of more efficient resource utilization, increased coordination of care and improved economic outcome. ${ }^{[14]}$ Much is needed in the health sectors in the present decade and many of the constraints cannot easily be resolved by money alone. In order to achieve goals in this aspect, health care providers who deliver the care at primary level will need to provide more complex intervention than has ever been expected of them. ${ }^{[13]}$ In a series of international meetings in the Global Forums on Health Research, health care providers repeatedly emerged as the most important constraint in responding to health care crises. ${ }^{[15]}$ Therefore, health care providers challenges, barriers, and readiness for different health care models and different consequences have always been a subject for discussion and exploration.

Furthermore, the strategic vision at the Ministry of Health in Oman, in collaboration with WHO experts, is continuously taking action and focusing attention on fighting diabetes and resolving the related challenges available in diabetes clinics in primary health care. Previous studies in this regard ${ }^{[16-18]}$ conducted for the Ministry of Health in Oman to improve diabetes management clinics at primary health care level, proposed that the main challenges at the diabetes clinics, which require immediate attention, were at the level of physicians' shortages, teams' models and workflow organization. Therefore, there is great need for this study to explore care providers' perceptions towards different models of care that could be applied within the available team-based approach in the clinics to solve some of the reported challenges and to explore the requirements for their implementation in diabetes 
clinics at primary health care in Muscat.

\section{METHOD}

\subsection{Study design}

This study was conducted along with other three studies that comprise a bigger project titled "Exploring the feasibility of interdisciplinary teams in the management of diabetes at primary health care level in Oman." This qualitative study was designed to include 27 semi-structured interviews with health care providers involved in diabetes care clinics at five purposively selected public primary health care centres in Muscat.

\subsection{Study setting}

The study was conducted in Muscat, the capital of Oman, which is one of the Gulf countries. The total population of Oman is 4,414,051 people and non-Omanis make up more than $40 \%$ of the present total population. ${ }^{[19]}$ Health care services in Muscat are provided through three different health care levels - primary, secondary and tertiary - and involve public and private institutes. Diabetes care in public primary health care in Muscat is provided in 26 out of 27 health care centres (one centre does not have the diabetes management resources to provide the service). In some centres, additional supportive members are added to the core team, such as pharmacists, assistant pharmacists, psychologists and medical orderlies. The diabetes clinics in primary health care, including service deliver and the team members' tasks and roles have been described elsewhere. ${ }^{[20]}$ Diabetes care in the private sector is delivered through a single physician approach. Therefore, the private sector was excluded from this study.

\subsection{Selected primary health care centres}

The selection of the five centres was performed simultaneously for the three qualitative studies in the project, including the current study. The other two studies are based on observations of diabetic patients and care providers during service provision $^{[20]}$ and interviews with patients. The selection was based on the results revealed from the first (quantitative) study of the project ${ }^{[8]}$ and aimed at maximum variation among the centres. The selected primary health care centres included: 1) a centre with mixed nationalities of health care providers on the diabetes care team; 2) a centre with only Omani health care providers on the diabetes care team; 3 ) a centre with no pharmaceutical and technical resources related to the diabetes care team; 4) a centre with absence of some of the health care providers from the diabetes care team; and 5) a centre with the highest number of diabetic patients recorded per centre in Muscat and the presence of additional health care providers to the diabetes care team.

\subsection{Characteristics of the participants}

A total of 27 health care providers who are involved in diabetes care team in the selected centres and were available at the time of conduction of the study were included. The included participants were core team members as follows: 11 physicians, eight nurses, two dieticians, and two health educators and supportive members as follows: a pharmacist, an assistant pharmacist, a psychologist and a medical orderly (a non-medical staff member who is responsible for service flow at the PHC centres) with a level of education ranged from postgraduate to specialized studies among all of them except for a medical orderly who held a basic education. The health care providers interviewed comprised twenty-six women and one man, with ages ranging between thirty and forty-five years. In addition to Omanis, the physicians interviewed were of mixed nationalities from different Asian and African countries; the other health care providers were all Omanis. The total years of experience for all health care providers in diabetes management ranged between five and twenty years.

\subsection{Data collection}

The data was collected through semi-structured interviews. The research team designed the interview protocol in advance and the first author conducted the interviews. The protocol involved questions related to different models of care in diabetes management clinics as follows:

- How could the official models of care that are already known (task-sharing, task-shifting, task-replacement) be considered as solutions to the shortage in physicians at diabetes management clinics? (Definitions of the three different models of care from the literature were provided for those who were not aware of them.)

- What are their opinions on the application of different models of care between members of the diabetes care team?

- What are the requirements and expected future outcomes of different models of care if implemented in diabetes care clinics in public primary health care centres in Oman?

Data collection started in January 2016 and ended in March 2016. One week was appointed for each of the five centres to complete collection of data (from Sunday to Thursday). The centre was visited every day in the morning and afternoon when required to see how the centre was operated and eventually to interview the participants. The health care providers were individually interviewed for 30-45 minutes in the clinic after concluding the service. The interviews were conducted in English or Arabic and audio recorded. 


\subsection{Data analysis}

Thematic analysis was applied and inspired by the steps described by Braun and Clarke. ${ }^{[21]}$ All the interviews were transcribed verbatim and translated to English if written in Arabic. The initial step involved familiarising with the data to obtain a sense of the whole, by reading through the transcripts repeatedly. The next step was the development of codes. The transcripts were entered into the Open Code computer program, ${ }^{[22]}$ which is designed to support qualitative data analysis. The analysis process was performed through an inductive approach where the codes were identified from data and the entire analysis was performed at the level of manifest qualitative data analysis. ${ }^{[21]}$ Words and sentences containing aspects related to each other were labelled with a code close to the text. The first author developed the initial codes. The initial codes were then shared with the second author for review. Codes with similar content were later grouped under initial sub-themes. The initial sub-themes were reviewed and refined to ensure the validity in relation to study aim and the data set. Finally the sub-themes were advanced into one main theme. ${ }^{[21,23]}$

\section{RESUlts}

The analysis resulted in one overarching theme: "Yes to discuss different models of care, but not to complete implementation yet." The theme captures the care providers' agreements towards task-sharing model within a team-based approach, but also their concern about complete implementation. The findings will be further presented in terms of four sub-themes: 1) Preferable task-sharing model; 2) Positive outcomes from task-sharing model; 3) Concerns during the change process; and 4) The requirements needed for the complete implementations of the task-sharing model.

\subsection{Preferable task-sharing model}

All health care providers expressed positive opinions towards changes in models of care at diabetes clinics. The health care providers opinions on task-sharing were more readily acknowledged and recognized compared to those on task-shifting and task-replacement. This was due to the fact that task-sharing was already performed to some extent between members of the team, such as physicians/nurses and dieticians/health educators. The task-sharing model between physicians and nurses involved enquiring about patients' complaints, performing examinations, and providing advice on healthy lifestyle habits. The task-sharing model between dieticians and health educators included discussion of a healthy lifestyle for Type 2 diabetes. Additionally, the physicians who had received training in other countries shared their experience and mentioned that they had seen task-sharing model being implemented with posi-

Published by Sciedu Press tive outcomes for the care providers, diabetes management clinics and patients. Moreover, the greater acceptance of task-sharing over the other models was due to the fact that in task-shifting and task-replacement, physicians would no longer be involved in the clinics and would leave the job completely to the other members of the team. Such action could not yet be accepted in primary health care centres in Muscat because the nurses are not yet fully equipped to handle the clinic independently, as revealed by the physicians and nurses.

"In other countries, and specifically where I was trained, I worked in an environment of task sharing and I was happy with the outcomes." (Physician)

"For us, I think sharing is already done, but for shifting and replacement, we are not on that yet, because we cannot work without the presence of the physician to refer to." (Nurse)

\subsection{Positive outcomes from task-sharing model}

The health care providers revealed that the task-sharing model within the team-based approach could contribute to many positive outcomes. For example, it was well known by the health care providers that task-sharing could contribute to proper time management and better patient management. For instance, patients with uncontrolled diabetes and those with comorbidities should be seen by physicians, but those with well-controlled diabetes could be seen by nurses. Consequently, the patients' outcomes will be improved, as the time spent with patients will be longer and the management will be more focused. An additional positive outcome that was mentioned was efficient service delivery at the clinic: for example, contribution of all the members of the team during service delivery on the same day, without far-flung appointments. Similarly, task-sharing could improve diabetes management visits by developing the roles of both care providers and diabetic patients and involving the latter more in the management of their condition. The nurses in this regard added that they could also act as practitioners, which would allow them to secure fixed jobs, associated with competent empowerment.

"The task-sharing is a good concept for time management for the physician and the patient. Also, the physicians' load could be reduced if well-controlled patients were transferred to the nurses, so the physicians could focus on patients who really require their management." (Dietician)

"The sharing concept speeds up the clinic and reduces the waiting time." (Health Educator) 
“This (task-sharing), if implemented completely, would provide better patient services, and consequently the patients would have better outcomes and we could secure fixed roles in the diabetes management clinics." (Nurse)

\subsection{Concerns during the change process}

The views expressed by the physicians towards complete application of task-sharing model were mixed, with some concerns related to the nurses. The physicians expressed their willingness to share their tasks with nurses. However, several concerns regarding nurses' knowledge and skills were anticipated and the nurses defined these worries as limitations and commented that their limitations in knowledge and skills in the context of diabetes management clinics are in terms of medication control, requesting patients' tests and following up patients with appropriate decisions.

"I am a trained staff (nurse) but I have not received any training regarding medication, follow-up on patients' results and making decisions regarding patients' management. I am not authorized to do these things, as I have not received enough training." (Nurse)

"I can ask them (patients) about their compliance with their medication and about their blood sugar control. I am not fully equipped to discuss the medication, but as for the other things, such as health education, examination and other computer work, I can do them." (Nurse)

The physicians in this regard expressed their sincere support towards developing and improving nurses' roles to be active team players. This was discussed in the context of sending the controlled patients to them and supporting them with follow-up visits. The physicians added that nurses would require time to overcome their weaknesses. Training program from the Ministry of Health and encouragement from nurses' managers, which would improve their knowledge and enhance their skills in the future, is needed.

"At the moment, we have patient categorization. We have categories A, B \& C. Category A includes the well-controlled patients and category $\mathrm{C}$ includes the very complicated patients. We can assign category $\mathrm{B}$ and $\mathrm{C}$ patients to the physician and $\mathrm{A}$ to the nurse. Patients who are very well controlled (A) can also be seen once a year by a physician and the nurse can conduct the follow-up visits, but at the moment, the nurses are not well equipped to follow up the well-controlled patients alone." (In-charge physician)

"If they (nurses) want to be diabetic nurses, and if they are given support, they can be very good in handling it (diabetes)." (Physician)

Further worries related to task-sharing came from nurses and were related to the diabetic patients. The nurses revealed that patients in the diabetes care clinics play a vital role and should be considered a very important factor for the success of team-based diabetes management. Therefore, complete implementation of task-sharing model within a team-based approach requires patients' agreement and acceptance. However, at the current stage, the nurses revealed that the patients could become angry because they are not ready to see the nurses independently in the clinic without physicians. The pharmacists added that the patients are not yet ready for complete application of the task-sharing model, but that they (patients) should help in the process of application, although this could only happen if the patients are ready. In this regard a comprehensive program is required that markets to patients the value of team-based care.

"Some of them (patients) will refuse to let the nurse examine them and do everything for them independently from the physician because they think that we are not qualified to do these things." (Nurse)

"The patients will not agree to be seen by nurses independently, especially patients from the older generations, but any change needs time." (Pharmacist)

"Patients should help us in applying the concept. They are sometimes angry with us when we try to help them, as they want the physician only to be involved. This cannot be applied now because patients are not ready." (Nurse)

\subsection{Requirements for full implementation of the task- sharing model}

The health care providers revealed that the changes in service delivery should be administered with careful examination of the requirements and step-wise application. Moreover, they added that the task-sharing model could be further developed between the physicians and the nurses. This view was favoured mainly because in the current diabetes management clinics in Muscat, physicians and nurses already share a room in the centre and provide their services in the same room. On the other hand, dieticians and health educators also share a room, but do not always provide their services in the same room. 
The health care providers discussed the requirements for application of the task-sharing model within the team-based approach extensively. Accordingly, they revealed that any change in the service organization is feasible. However, it has to start from high authorities in the ministry. Also, they added that models of care in the centres require clear policy, clear criteria for the health care providers, reviewed job descriptions, training, and appropriate follow-up from the stakeholders at the ministry. The physicians disclosed that if the concern is related to the nurses, then clear revision of their roles is mandatory, and added that for the task-sharing model to be fully implemented, higher authority involvement is required.

"We need clear criteria as to who does what and when, otherwise it (task sharing) will not work." (Nurse)

"If we could see training and proper follow-up in their (nurses) job descriptions and in their policies, we could do it (task-sharing), but it is not there. So now we cannot apply it." (In-charge physician)

"Somebody from the higher authority should look at proper training of nurses. We want to get a nurse who can prescribe medication: we have been working on this, but there is still resistance from the higher authorities and I am not sure why." (In-charge physician)

The care providers also expressed their clear need for communication with the patients in order to accommodate the nurses' presence in the clinic differently from what they are used to. This communication was mentioned in relation to patients' strong attachments to the Omani culture and their usual clinical habits (seeing a physician in the clinic).

"In order to accommodate patients within the new concept, we have to clearly communicate and explain the concept to them, and associate it with the Omani culture." (Physician)

The study revealed that trust between the health care providers themselves and between them and the patients is vital, and should thus be an important requirement for the implementation of sharing model.

"The sharing concept requires trust between the patients and the providers, especially the nurses." (Physician)

"Nothing will work if the patients do not agree, and nothing will convince them unless they start to trust us." (Nurse)
With regard to trust, the pharmacists supported the tasksharing model between themselves and the physicians, and added that a physician/pharmacist sharing model could help in terms of medication management. They added that physician/nurse task-sharing is also feasible, but not yet in medication management, as nurses could only be involved with medication if they had undergone appropriate training.

"At the moment, I think I can trust only the physician with the medication and we can make a good pair, but some training for nurses could empower them too" (Pharmacist)

\section{Discussion}

The study presents task-sharing within team-based approach as the most suitable and preferable model of care according to the health care providers. They revealed that it is already done to a certain degree and is mainly applied between physicians and nurses. The study also explores some concerns towards the mechanism and offers some requirements for its complete implementation.

The providers disclosed that a task-sharing model with proper implementation could improve diabetes management clinics and that this could be comprehended in terms of better time management, reduction in physicians' load, and improvement in patients' outcomes. The literature in this regard supports physician/nurse task-sharing and promotes this strategy for improvement of patients' outcomes. ${ }^{[24-26]}$ Furthermore, the literature stresses that with proper models of care for workflow in health care and close relationships among colleagues, a team-based approach to diabetes management clinics can be established. ${ }^{[27]}$ The team-based approach is already known in Oman, but could move clinics to a higher level of nurses' independence, particularly given that the physicians believed that nurses' development through training and close monitoring could promote nurse-led clinics. The nurses, from their sides, expressed their willingness to be involved in the suggested change and looked at it as a fixed job opportunity and an improvement in patient-provider trust.

Several factors were discussed as concerns that needed to be addressed before the selected model could be fully implemented: primarily readiness of the providers and patients' acceptance. The providers' involvement in this study represents the main core of the diabetes management team (the four members) and the supportive members in some centres. Although the physicians were of mixed nationalities, all of them revealed the same concerns towards the nurses and reservations towards allowing them to perform certain clinical tasks. The weaknesses were found to be in clinical 
decision-making and medication management. The nurses confirmed their weaknesses in certain tasks and explained this in relation to their basic training. The literature highlights this issue by exploring the gaining of knowledge and insight into managerial and leadership skills and when this can be achieved, and shows that the required decision-making abilities are beyond nurses' current sphere of knowledge. ${ }^{[28]}$ Moreover, the literature also indicates that practicing nurses specifically feel that they do not have the time, access or expertise to search and analyse the research literature to answer clinical questions. ${ }^{[28]}$ Such questions, which include critical decision-making, might also include medication management, both of which are considered a challenge for nurses. Therefore, these weaknesses that were identified in this study were also reported in the literature, and it should be added that the majority of practicing nurses in clinical settings have less than a baccalaureate degree and may not have been exposed to formal research literature. Furthermore, the incompetent knowledge and skills which were found in our setting and confirmed by a previous study in this project ${ }^{[8]}$ contributed to the nurses' weaknesses and needs for their training to be updated and revised.

Although the nurses did not comment explicitly on the weaknesses in their knowledge and skills, patients' nonacceptance of task-sharing mechanisms was their main concern and was described as a difficult subject but an important factor for discussion in diabetes management clinics. The study revealed that nurses had high expectations of patients anger and non-acceptance towards them. This might be because all the nurses interviewed were Omanis and were thus aware of the Omani culture and the community. In this regard, the literature supports our findings and describes patients' anger towards nurses as a common attitude and a longstanding problem in many countries, ${ }^{[29,30]}$ which requires serious attention to help nurses to cope with such an approach. Consequently, nurses' mechanisms for coping with aggressive patients' attitudes require further development. ${ }^{[31]}$ In this study, the patients' anger and non- acceptance could also be considered as a consequence of the nurses' weak knowledge and skills and both perspectives could be understood, but further research is required before solutions can be proposed. In this regard, patients require an effective campaign to help them to understand that team-based care is the optimal care.

The health care providers mentioned several requirements for complete implementation of task-sharing model within a team-based approach, but strongly suggested support for nurses from higher authorities, namely Ministry of Health authorities and centre managers. Further to these requirements, they revealed that some of the pre-requisites for task-sharing implementation are already available in the clinics and might help in preparing them further for nurse-led clinics. These pre-requisites were mentioned in terms of categorization of diabetic patients and the availability of supportive physicians to enable nurses to apply their knowledge with close and guided supervision. The physicians in this study were very supportive and had positive attitudes towards the nurses, and were in favour of nurses' role improvement, which was considered a very important factor for the nurses' retention and perseverance. ${ }^{[32]}$ This support was mentioned in terms of appropriate training, clear criteria and clear definition of tasks for the nurses. In fact, in this regard, nurses with good training have been described in the literature as a motivator for improvement of clinic outcomes, ${ }^{[33]}$ and in this study, were described by the physicians as good helpers for the team.

The Omani culture could play a major role in the process of implementation of the model of care, as patients' compliance to the process depends a lot on their understanding and acceptance to the process within the context of Omani culture. This acceptance will not be easy unless the health care providers enhance behavioural change in diabetic patients and provide evidence that better health care delivery systems and reforms can improve their accessibility, and efficiency of care and are likely to help primary health care centres to meet international standards of care. ${ }^{[34]} \mathrm{A}$ framework of cultural competence barriers can be seen at different levels, but proper education and communication with patients on cross-cultural issues could enhance health and health care. ${ }^{[35]}$ Similarly, the literature reveals that with particular chronic conditions, the nurses, more than physicians, are primarily responsible for first contact care for patients, and that patients' satisfaction is higher with nurse-led care because nurses tend to provide longer consultations, give more information to patients and recall patients more frequently than do physicians. ${ }^{[36]}$ These factors could provide additional support and help to enable patients to understand the reasons behind the implementation of task-sharing. The care providers in this study also revealed that improving patients' attitudes requires time and marketing. Furthermore, the professional application of the skills and sharing model of care from the nurses' side requires attention.

\section{Methodological considerations}

One of the limitations of this study was the unequal gender distribution among the providers: therefore, we could not build our analysis on gender. The gender imbalance could be explained in relation to the higher number of female providers compared to their male counterparts at primary health care centres. ${ }^{[8]}$ The health care providers who were of mixed nationalities (physicians) provided more perceptions 
and feedback towards the models of care compared to other providers, who were all Omanis, and this could be explained by experiences from their countries of origin and by the Omani physicians' specialization, which was gained from abroad. The interviews were conducted in a professional way, with maximum research ability, despite the hierarchical perception towards physicians at Ministry of Health, and all providers were given voices and chances to express their perceptions equally, which gave them a sense of importance and validity in the diabetes management team. The study setting was only in Muscat and specifically in five primary health care centres: a situation that limits the transferability of the findings to other regions. However, as transferability was not the intention, the exploration of providers' perceptions of the models of care was a valuable contribution to the entire project.

The first author, who is a physician in Ministry of Health, acted as an insider and totally understood the perception of physicians in this study, who wanted to move the clinics to a higher level. At the same time, the same researcher acted as an outsider because she does not work in a diabetes management clinic, which allowed her to understand the perspectives of other professional groups as well.
The negative aspect of being an insider (a physician in the Ministry of Health) is being part of the ministry hierarchical system. However, this concept was avoided during the running of this study and appropriate space was given to all the providers. The negative side of being an outsider is the practicality of the implementation of nurse-led clinics and the extent to which the challenges encountered in the diabetes management clinics could be solved.

\section{Conclusion}

According to health care providers, a task-sharing model of care within a team-based approach was considered the appropriate model for implementation in diabetes management clinics in the future. However, the requirements for this task-sharing model must first be prepared and extensively followed up by the ministry and health care providers, and must be supported by the diabetic patients. The process will require time, effort and patients' understanding of the model, which could eventually lead to nurse-led clinics within a team-based approach and therefore should be taken very seriously and considered carefully.

\section{CONFLicts OF INTEREST Disclosure}

The authors declare no conflicts of interest.

\section{REFERENCES}

[1] Callaghan M, Ford N, Schneider H. A systematic review of taskshifting for HIV treatment and care in Africa. Human Resources for Health. 2010; 8(1): 8. PMid:20356363. https://doi.org/10.1 186/1478-4491-8-8

[2] Joshi R, Alim M, Kengne AP, et al. Task shifting for noncommunicable disease management in low and middle income countries-a systematic review. 2014; 9(8): e103754. PMid:25121789. https://doi.org/10.1371/journal.pone.0103754

[3] Mullan F, Frehywot S. Non-physician clinicians in 47 sub-Saharan African countries. The Lancet. 2007; 370(9605): 2158-63. https : //doi.org/10.1016/S0140-6736(07)60785-5

[4] Laurant M, Reeves D, Hermens R, et al. Substitution of doctors by nurses in primary care. The Cochrane Library. 2005. PMid:15846614. https://doi.org/10.1002/14651858.CD001271.pub2

[5] WHO. Task shifting: rational redistribution of tasks among health workforce teams: global recommendations and guidelines. 2007.

[6] WMA. Resolution on task shifting from the medical profession. New Delhi. 2009

[7] Islam SMS. Human Resources for Non-Communicable Diseases in Bangladesh. International Journal of Perceptions in Public Health. 2017; 1(2): 98-101.

[8] Al-Alawi K, Johansson H, Al-Mandhari A, et al. Are the resources adoptive for conducting team-based diabetes management clinics? An explorative study at primary health care centers in Muscat, Oman. Primary Health Care Research \& Development. 2018; 1-28. PMid:29737963. https://doi.org/10.1017/S1463423618000 282

Published by Sciedu Press
[9] IDF. Global Guideline for Type 2 Diabetes [Clinical Guidelines Task Force]. 2012. Available from: http://www.idf .org/sites/de fault/files/IDFT2DMGuideline.pdf

[10] IDF. The IDF Diabetes Atlas 2015 [cited 2016 August 23]. Available from: http://www.idf.org/diabetesatlas

[11] Handelsman Y, Bloomgarden ZT, Grunberger G, et al. American Association of Clinical Endocrinologists and American College of Endocrinology-clinical practice guidelines for developing a diabetes mellitus comprehensive care plan-2015. Endocrine Practice. 2015; 21(s1): $1-87$

[12] Anand S, Bärnighausen T. Human resources and health outcomes: cross-country econometric study. The Lancet. 2004; 364(9445): 1603 9. https://doi.org/10.1016/S0140-6736(04) 17313-3

[13] Hongoro C, McPake B. How to bridge the gap in human resources for health. The Lancet. 2004; 364(9443): 1451-6. https ://doi .or $\mathrm{g} / 10.1016 / \mathrm{S} 0140-6736$ (04) 17229-2

[14] Mitchell P, Wynia M, Golden R, et al. Core principles \& values of effective team-based health care. Institute of Medicine Washington, DC; 2012. https://doi.org/10.31478/201210c

[15] Narasimhan V, Brown H, Pablos-Mendez A, et al. Responding to the global human resources crisis. The Lancet. 2004; 363(9419): 146972. https://doi .org/10.1016/S0140-6736(04) 16108-4

[16] Abdulhadi N, Al-Shafaee M, Freudenthal S, et al. Patient-provider interaction from the perspectives of Type 2 diabetes patients in Muscat, Oman: a qualitative study. BMC Health Services Research 2007;7(1):162. PMid:17925030. https://doi .org/10.1186/14 72-6963-7-162 
[17] Abdulhadi N, Al-Shafaee MA, Östenson CG, et al. Quality of interaction between primary health-care providers and patients with type 2 diabetes in Muscat, Oman: an observational study. BMC Family Practice. 2006; 7(1): 72. PMid:17156424. https ://doi .org/10 .1186/1471-2296-7-72

[18] Abdulhadi NMN, Al-Shafaee MA, Wahlström R, et al. Doctors' and nurses' views on patient care for Type 2 diabetes: an interview study in primary health care in Oman. Primary Health Care Research \& Development. 2013; 14(03): 258-69. PMid:23259934. https://doi.org/10.1017/S146342361200062X

[19] MOH. Annual Health Report. Muscat: Ministry of Health, Department of Health Information and Statistics; 2016.

[20] Al-Alawi K, Al-Mandhari A, Johansson H. Care providers' perceptions towards challenges and opportunities for service improvement at diabetes management clinics in public primary health care in Muscat, Oman: a qualitative study. BMC Health Services Research. 2019; 1(19): 1-18. PMid:30621675. https://doi.org/10.1186/s129 13-019-3866-y

[21] Braun V, Clarke V. Using thematic analysis in psychology. Qualitative Research in Psychology. 2006; 3(2): 77-101. https://doi .or $\mathrm{g} / 10.1191 / 1478088706 \mathrm{qp} 063$ oa

[22] The Open Code Computer Programme. Available from: http: //www.umu.se/phmed/epidemi/forskning/open_code.html

[23] Smith JA. Qualitative psychology: A practical guide to research methods: Sage; 2015.

[24] Way D, Jones L, Busing N. Implementation strategies: collaboration in primary care - family doctors \& nurse practitioners delivering shared care. Toronto: Ontario College of Family Physicians. 2000; 8.

[25] Zwarenstein M, Bryant W. Interventions to promote collaboration between nurses and doctors. Cochrane Database Syst Rev. 2000; 2 : CD000072. https://doi.org/10.1002/14651858.CD000072

[26] Tang C, Chan S, Zhou W, et al. Collaboration between hospital physicians and nurses: an integrated literature review. International Nursing Review. 2013; 60(3): 291-302. PMid:23961790. https://doi.org/10.1111/inr.12034
[27] Beach MC, Inui T, Network RCCR. Relationship-centered care: A constructive reframing. Journal of General Internal Medicine. 2006; 21(S1): S3-S8. PMid:16405707. https://doi.org/10.1111/j . 1525-1497.2006.00302.x

[28] Marquis BL, Huston CJ. Leadership roles and management functions in nursing: Theory and application. Lippincott Williams \& Wilkins; 2009.

[29] Holman H, Lorig K. Patients as partners in managing chronic disease: partnership is a prerequisite for effective and efficient health care. British Medical Journal. 2000; 320(7234): 526. PMid:10688539. https://doi.org/10.1136/bmj.320.7234.526

[30] Needham I, Abderhalden C, Halfens RJ, et al. Non-somatic effects of patient aggression on nurses: a systematic review. Journal of Advanced Nursing. 2005; 49(3): 283-96. PMid:15660553. https://doi.org/10.1111/j.1365-2648.2004.03286.x

[31] Bailey RD, Clarke M. Stress and coping in nursing. Springer; 2013.

[32] Rosenstein AH. Nurse-physician relationships: impact on nurse satisfaction and retention. The American Journal of Nursing. 2002; 102(6): 26-34. PMid:12394075. https : //doi .org/10.1097/00 000446-200206000-00040

[33] Bodenheimer T, MacGregor K, Stothart N. Nurses as leaders in chronic care. British Medical Journal Publishing Group; 2005. PMid:15774966. https ://doi .org/10.1136/bmj.330.7492.6 12

[34] Chin MH, Cook S, Jin L, et al. Barriers to providing diabetes care in community health centers. Diabetes Care. 2001; 24(2): 268-74. PMid:11213877. https://doi.org/10.2337/diacare.24.2.2 68

[35] Betancourt JR, Green AR, Carrillo JE, et al. Defining cultural competence: a practical framework for addressing racial/ethnic disparities in health and health care. Public Health Reports. 2016.

[36] Laurant M, Reeves D, Hermens R, et al. Substitution of doctors by nurses in primary care. Cochrane Database Systematic Review. 2005; 2: CD001271. PMid:15846614. https ://doi.org/10.1002/14 651858. CD001271. pub2 